\title{
Nutritional approach to failure to thrive
}

Su lin Jeong, $M D$

Department of Pediatrics, CHA Bundang Medical Center, CHA University, Seongnam, Korea

Received: 16 May 2011, Accepted: 27 May 2011

Corresponding author: Su Jin Jeong, MD

Department of Pediatrics, CHA Bundang Medical Center, CHA University, 351 Yatap-dong, Bundang-gu, Seongnam 463-712, Korea

Tel: +82-31-780-5230, Fax: +82-31-780-5239

E-mail: jinped@cha.ac.kr

Copyright $(\odot 2011$ by The Korean Pediatric Society
Failure to thrive (FTT) is a term generally used to describe an infant or child whose current weight or rate of weight gains is significantly below that expected of similar children of the same age, sex and ethnicity. It usually describes infants in whom linear growth and head circumference are either not affected, or are affected to a lesser degree than weight. FTT is a common problem, usually recognized within the first 1-2 years of life, but may present at any time in childhood. Most cases of failure to thrive involve inadequate caloric intake caused by behavioral or psychosocial issues. The most important part of the outpatient evaluation is obtaining an accurate account of a child's eating habits and caloric intake. Routine laboratory testing rarely identifies a cause and is not generally recommended. FTT, its evaluation, and its therapeutic interventions are best approached by a multi-disciplinary team includes a nutritionist, a physical therapist, a psychologist and a gastroenterologist. Long term sequelae involving all areas of growth, behavior and development may be seen in children suffering from FTT. Early detection and early intervention by a multidisciplinary team will minimize its long term disadvantage. Appropriate nutritional counseling and anticipatory guidance at each well child visit may help prevent some cause of FTT.

Key words: Failure to thrive, Inadequate caloric intake, Nutritional counseling, Multi-disciplinary team

This is an open-access article distributed under the terms of the Creative Commons Attribution Non-Commercial License (http://creativecommons.org/licenses/by$\mathrm{nc} / 3.0 /$ ) which permits unrestricted non-commercial use, distribution, and reproduction in any medium, provided the original work is properly cited.

\section{Introduction}

There is no consensus on which specific anthropometric criteria should be used to define failure to thrive $(\mathrm{FTT})^{1-4)}$. In routine clinical practice, the three weight criteria that can be used to described FTT are a child younger than 2 years whose weight is below the $3 \mathrm{rd}$ or 5 th percentile for age on more than one occasion, a child younger than 2 years whose weight is less than $80 \%$ of the ideal weight for age, and a child younger than 2 years whose weight crosses two major percentiles downward in a standardized growth grid using the 90th, 75th, 50th, 25th, 10th, and 5th percentiles as the major percentiles ${ }^{5}$.
A combination of anthropometric criteria, rather than one criterion, should be used to more accurately identify children at risk of FTT. Weight for length is a better indicator of acute under-nutrition and is helpful in identifying children who need prompt nutritional treatment ${ }^{6}$. This is an index of fat stores that defines whether a patient suffers from acute malnutrition. Any weight change below the 5 th percentile may indicate a child is at risk of $\mathrm{FTT}^{7}$. Finally, some children who falter in growth parameters actually demonstrate a normal variant of growth, such as children of small parents who are growing to their full genetic potential, large for gestational age infants who regress toward the mean, children with constitutional delay in 
growth, or premature infants whose growth parameters are normal when corrected for gestational age ${ }^{8}$. When uncertain, a weight for age that falls below the 5 th percentile or a weight deceleration that crosses two major percentile lines should prompt the use of additional growth indices, such as weight for length or weight velocities, to confirm the growth trend.

\section{Prevalence}

Most of the time, insufficient growth is detected in a routine visit to the pediatrician or the family practitioner. Many parents do not seem to notice the situation until it is brought to their attention. In most instances, FTT is insidious and gradual. It can be missed by a carefully maintained growth chart.

Estimates of the incidence of FTT vary widely depending on the terminology and the population studied:

- Growth deficiency affects $10 \%$ of the rural outpatient population. The same percentage applies to high risk groups such as the homeless".

- FTT accounts for 1 to $5 \%$ of pediatric hospital admission for children younger than 2 years in the United State. National and state surveys indicate that as many as $10 \%$ of the children seen in primary care practice have signs suggesting inadequate growth ${ }^{5}$.

- In inner city emergency department, approximately 15 to $30 \%$ of young children who receive acute care services show signs of growth deficits ${ }^{5}$.

- Up to 15 to $20 \%$ of hospitalized children younger than 2 years from a medically indigent population met the criteria of $\mathrm{FTT}^{5)}$.

\section{Etiology}

Failure to thrive may result from a variety of "organic" and "nonorganic" causes for a combination of both. In evaluation of a child with FTT, the diagnostic efforts should be directed toward determining whether the child has one or a combination of the following factors contributing to the etiology: inadequate caloric intake, inadequate caloric absorption, or excessive caloric expenditure $^{8,10)}$. There is increasing recognition that in many children the cause is multi-factorial and includes biologic, psychosocial and environmental contributors ${ }^{11}$. Furthermore, in more than 80 percent of cases, a clear underlying medical condition is never identified. Table 1 provides a differential diagnosis of FTT. In adequate caloric intake is the most common etiology seen in primary care settings. In infants younger than eight weeks, problems with feeding (e.g., poor sucking and swallowing) and breast feeding difficulties are prominent ${ }^{12}$. After then, transitioning to solid foods, insufficient breast milk or formula consumption, excessive juice consumption, and parental avoidance of high-calorie foods often lead to FTT $^{13)}$.

Family factors can contribute to inadequate caloric intake at any age. These include mental health disorders, inadequate nutritional knowledge, and financial difficulties. Poverty is the greatest single risk factor for FTT in developed and developing countries. Importantly, child neglect of abuse must be considered, because children with FTT are four times more likely to be abused than children without FTT $^{14)}$.

Inadequate caloric absorption includes disorders causing frequent emesis (e.g., metabolic disorders, food insensitivities) or malabsorption (e.g., chronic diarrhea, protein losing enteropathy). Excessive caloric expenditure usually occurs in the setting of a chronic condition, such as congenital heart disease, chronic pulmonary disease, or hyperthyroidism. In these instances, FTT often develops during the first eight weeks of life.

\section{Clinical approach to failure to thrive}

The parents often do not recognize the subtle slowing of growth in their child in close day to day living. The family and the child's caregiver should be carefully evaluated with a thorough review

Table 1. Pathological Causes of Failure to Thrive

Inadequate food intake
Lack of appetite
Chronic illness or anemia
Psychosocial disorder
Food not available
Type or volume of food not appropriate
Feeding technique, parental-infant interaction problems
Withholding of food
Reduced absorption or digestion of nutrients
Pancreatic insufficiency
Loss of damage to callous surface
Excessive loss of nutrients
Vomiting
Gastro-intestinal : gastro-esophageal reflux, obstructions

Gastro-intestinal : gastro-esophageal reflux, obstructions
Central nervous system causes: increased intracranial pressure, drugs

Systemic illness: urinary tract infection or other infection, metabolic disorders

Malabsorption/diarrhea Inflammatory bowel disease. Pancreatic insufficiency, colitis

Renal losses

Renal failure / renal tubular acidosis

Diabetes mellitus or diabetes insipidus

Inability to properly utilize ingested nutrients

Chromosomal or genetic abnormality

Metabolic disorder

Endocrine disorder

Excessive utilization of energy

Chronic illness (e.g.. cardiac disease, liver/renal failure, endocrine disorders, infection, anemia) 
of family history. Thus, history taking is the most important investigative technique in the evaluation of FTT. The differential diagnosis is vast, but in most cases, there are sufficient symptoms and signs that lead to a specific diagnosis. The following data should be included in the history ${ }^{6}$.

- Feeding: breast feeding or formula feeding, formula preparation, volume consumed, who feeds the infants, position and placement of the infant for feeding, timing and introduction of solids, stool or vomiting patterns associated with feeding, strength of suck. Accurate measurement of caloric intake of infants and children should be done by proper diet history.

- Developmental history: gestational and perinatal history (age and parity of mother, medical complication of pregnancy, use of medications, use of drugs, use of alcohol, substance abuse, smoking, complications during delivery), developmental milestones, temperament.

- There may be specific behavior characteristics of the child that play an important role. These characteristics include disrupted sleeping and eating patterns and behavior that is moody, demanding, rejecting, or distractible. Some authors have suggested that a subset of infants may refuse to eat in an attempt to achieve some degree of autonomy and control over their mothers and have termed this infantile anorexia nervosa.

- Psychosocial history: family composition, employment and financial status, stress, potential isolation, child rearing beliefs, history of maternal depression, the caretaker's history of childhood neglect or abuse.

- Family history: heights, weights, illness, development that may indicate constitutional short stature, inherited disease, developmental delay.

It is important to observe the infant and caretaker while feeding and playing. This provides clues about their interactions, the caretaker's feeding technique, or inappropriate response to the infant's physiologic or social cues. The infant may avoid eye contact or withdraw from physical attention or may show poor suck or swallow or aversion to oral stimulation.

\section{Physical examination}

A complete and carefully physical examination is essential. Plot the patient's weight, recumbent length in infants younger than 2 years, and the head circumference on a standard growth curve specific for gender. This information helps to narrow the etiology of FTT.

- Head circumference, weight and height are proportionately reduced in infants and children who have hereditary and congenital defects.
Table 2. Red Flag Signs and Symptoms Suggesting Medical Causes of Failure to Thrive

Cardiac findings suggesting congenital heart disease or heart failure (e.g., murmur, edema, jugular venous distention)

Developmental delay

Dysmorphic feature

Failure to gain weight despite adequate caloric intake

Organomegaly or lymphadenopathy

Recurrent or severe respiratory, mucocutaneous, or urinary infection

Recurrent vomiting, diarrhea, or dehydration

- Head circumference is normal and weight is slightly reduced proportionate to height in children with constitutional growth delay, genetic dwarfism or endocrinologic disorders.

- Head circumference is normal and weight is reduced out of proportion to height in most infants with FTT, especially if malnutrition results from inadequate caloric intake, malabsorption, or altered metabolism.

Other decisions the physician must make after examination is whether to admit the child to the hospital. Such decisions may be influenced if abuse or neglect is suspected; if the caretaker is psychosocially impaired; or if serious malnutrition (60\% below ideal body weight $)^{15)}$, traumatic injury, hypothermia, low pulse rate, or low blood pressure is present. Physicians should also seek red flag signs or symptoms of medical conditions that might be causing FTT ${ }^{13)}$ (Table 2).

Further examination beyond growth parameters should include a thorough general examination including inspection for any physical signs of neglect or abuse, dysmorphic features, skin rashes, examination of the mouth for the presence of a cleft palate and quality of sucking movements. The chest reveals signs of chronic respiratory or cardiac disease. The abdomen may be distended due to malabsorption, or there may be organomegaly. Observe the child's general appearance and look for body fat and muscle wasting. Examination of skin, hair, and mails is in valuable.

\section{Investigations}

Investigation should be guided by the history and examination. Infants who are either unwell or have significant positive physical findings will require immediate investigation and consideration of pediatric referral, while those who are generally well with no positive findings may no immediate investigations ${ }^{16)}$. In those requiring investigation, full blood examination, erythrocyte sedimentation rate, electrolytes including urea, creatinine, calcium and magnesium, and urine for culture and urinalysis are helpfully as an initial group of screening tests. If there are specific concerns raised in the history, other investigation may be indicated. In children where significant 
doubt persists and tests have been unhelpful, pediatric review and sometimes a period of hospitalization for a trial of observed feeding and further investigation may be helpful ${ }^{17)}$.

\section{Management}

FTT has serious implications, especially for brain development. If malnutrition becomes severe and chronic during the first year of life, the child's neurologic development may be permanently affected, making early recognition and prompt intervention critical.

A multidisciplinary team approach is essential for management, with the involvement of a pediatric gastroenterologist, a nutritionist, a social worker, an occupational therapist, a speech and physical therapist, a psychologist and behavioral and developmental specialists $^{18)}$.

The goals of management of FTT are following:

- Provision of adequate calories, protein, and other nutrients

- Nutritional counseling to the family

- Monitoring of growth and nutritional status

- Specific treatment of complications or deficiencies

- Long term monitoring and follow up

- Education of the family on social land nurturing techniques

- Supportive economic assistance

Whenever possible, the underlying cause of FTT should be addressed and treated. However, caloric supplementation is the major contributor to the management of FTT. Nutritional requirements can be assessed with the use of a formula for calories and protein $^{19)}$.

Catch -up growth requirement $(\mathrm{kcal} / \mathrm{kg} / \mathrm{d})=$ [calories required for age $(\mathrm{kcal} / \mathrm{kg} / \mathrm{d}) \times$ ideal weight for age $(\mathrm{kg})] /[$ actual weight $(\mathrm{kg})]$

If a diagnosis of FTT is made and no medical conditions are suggested on examination, appropriate guidance for catch-up growth should be made. Age appropriate nutritional counseling should be provided to parents ${ }^{16,17,20,21)}$. For parents of breastfed infants, recommending breastfeeding more often, ensuring lactation support, or discussing formula supplementation until catch up growth is achieved may helpful ${ }^{17}$. Parents of formula fed infants may be instructed on how to make energy dense formula by concentrating the ratio of formula to water during periods of catch up growth ${ }^{20,211}$.

The child's diet must be fortified for caloric density by using concentrated formula or by adding glucose polymers or extra lipids. Toddlers should be offered solid food before liquids and avoid excessive juice or milk consumption because this can interfere with proper nutrition ${ }^{22)}$. Nutritional supplements may be given until catch up growth is achieved. During period of catch up growth, parents may also be instructed to provide calorie dense foods such as peanut butter, cheese, dried fruits, and cream sauces for older children or adolescents. A supplement with a multivitamin with additional specific nutrients is recommended. The rule of 3's is quite helpful - 3 meals, 3 snacks, and 3 choices ${ }^{23)}$.

If a disease or medical condition is identified on history, physical examination, or additional testing, the correct approach will vary depending on the condition. Appropriate management may include instituting specific treatment of the condition, or seeking consultation from a subspecialist or other health care professional for further evaluation and management recommendations. Enteral tube feedings or gastrostomy should be considered for patients in whom oral enteral feeding dose not achieve adequate caloric intake.

Refeeding should proceed cautiously, because significantly undernourished children may develop diarrhea, vomiting, and circulatory decompensation ${ }^{24)}$.

Close follow up should be performed in the physician's office, including evaluation of height and weight. Multi-disciplinary interventions should be considered to improve weight gain, parent child relationships, and cognitive development. The catch-up height will lag several months behind the catch-up weight. Thus the nutritional intervention should be until appropriate height for age is reached.

Finally, although medications such as megestrol (Megace) or cyproheptadine have been shown to help promote weight gain in children with cancer related cachexia, they have not been studied in other causes of $\mathrm{FTT}^{25}$. Growth hormone therapy also has not been widely studied in children and adolescents who are not growth hormone deficient and is not recommended for management of FTT $^{26)}$.

\section{Prognosis}

There is consensus that severe, prolonged malnutrition, which is common in developing countries, can negatively affect a child's future growth and cognitive development ${ }^{27)}$. A history of FTT was associated with short stature, poor math performance, and poor work habits. A systemic review showed that FTT during the first two years of life was not associated with a significant reduction in intelligence quotient, although some long term reductions in weight and height were present ${ }^{28)}$. The possibility of long term cognitive and behavioral sequelae is present. Lastly, child with a history of FTT are at increased risk of recurrent FTT, and their growth should be monitored closely.

\section{References}

1. Olsen EM, Petersen J, Skovgaard AM, Weile B, Jørgensen T, Wright CM. Failure to thrive: the prevalence and concurrence of anthropometric 
criteria in a general infant population. Arch Dis Child 2007;92:109-14.

2. Jolley CD. Failure to thrive. Curr Probl Pediatr Adolesc Health Care 2003;33:183-206.

3. de Onis M, Garza C, Onyango AW, Borghi E. Comparison of the WHO child growth standards and the CDC 2000 growth charts. J Nutr 2007;137:144-8.

4. Olsen EM. Failure to thrive: still a problem of definition. Clin Pediatr (Phila) 2006;45:1-6.

5. Zenel JA Jr. Failure to thrive: a general pediatrician's perspective. Pediatr Rev 1997;18:371-8.

6. Shah MD. Failure to thrive in children. J Clin Gastroenterol 2002;35:3714.

7. Roche AF, Sun SS. Human growth: assessment and interpretation. Cambridge (UK): Cambridge University Press, 2003.

8. Bergman P, Graham J. An approach to "failure to thrive". Aust Fam Physician 2005;34:725-9.

9. Bithoney WG, Dubowitz H, Egan H. Failure to thrive/growth deficiency. Pediatr Rev 1992;13:453-60.

10. Wright CM, Parkinson KN, Shipton D, Drewett RF. How do toddler eating problems relate to their eating behavior, food preferences, and growth? Pediatrics 2007;120:e1069-75.

11. Emond A, Drewett R, Blair P, Emmett P. Postnatal factors associated with failure to thrive in term infants in the Avon Longitudinal Study of Parents and Children. Arch Dis Child 2007;92:115-9.

12. McDougall P, Drewett RF, Hungin AP, Wright CM. The detection of early weight faltering at the 6-8-week check and its association with family factors, feeding and behavioural development. Arch Dis Child 2009;94:549-52.

13. Cole SZ, Lanham JS. Failure to thrive: an update. Am Fam Physician 2011;83:829-34.

14. Wright CM. Identification and management of failure to thrive: a community perspective. Arch Dis Child 2000;82:5-9.

15. Gómez F, Ramos Galvan R, Frenk S, Cravioto Muñoz J, Chávez R, Vázquez J. Mortality in second and third degree malnutrition. 1956. Bull World Health Organ 2000;78:1275-80.
16. Careaga MG, Kerner JA Jr. A gastroenterologist's approach to failure to thrive. Pediatr Ann 2000;29:558-67.

17. Hren I, Mis NF, Brecelj J, Campa AS, Sedmak M, Krzisnik C, et al. Effects of formula supplementation in breast-fed infants with failure to thrive. Pediatr Int 2009;51:346-51.

18. Showers J, Mandelkorn R, Coury DL, McCleery J. Nonorganic failure to thrive: identification and intervention. J Pediatr Nurs 1986;1:240-6.

19. Rathbun JM, Peterson KE. Nutrition in failure to thrive. In: Grand RJ, Sutphen JL, Dietz WH, editors. Bosron: Butterworths, 1987;629-43.

20. Khoshoo V, Reifen R. Use of energy-dense formula for treating infants with non-organic failure to thrive. Eur J Clin Nutr 2002;56:921-4.

21. Clarke SE, Evans S, Macdonald A, Davies P, Booth IW. Randomized comparison of a nutrient-dense formula with an energy-supplemented formula for infants with faltering growth. J Hum Nutr Diet 2007;20:32939.

22. Sullivan PB, Goulet O. Growth faltering: how to catch up? Eur J Clin Nutr 2010;64 Suppl 1:S1.

23. Bauchner H. Failure to thrive. In: Kliegman RM, Behrman RE, Jeason HB, Stenton BF, editors. Nelson textbook of pediatrics. 18th ed. Philadelphia: Saunders, 2007:184-6.

24. Frank DA, Zeisel SH. Failure to thrive. Pediatr Clin North Am 1988;35: 1187-206.

25. Couluris M, Mayer JL, Freyer DR, Sandler E, Xu P, Krischer JP. The effect of cyproheptadine hydrochloride (periactin) and megestrol acetate (megace) on weight in children with cancer/treatment-related cachexia. J Pediatr Hematol Oncol 2008;30:791-7.

26. Sandberg DE. Should short children who are not deficient in growth hormone be treated? West J Med 2000;172:186-9.

27. Rudolf MC, Logan $S$. What is the long term outcome for children who fail to thrive? A systematic review. Arch Dis Child 2005;90:925-31.

28. Black MM, Dubowitz H, Krishnakumar A, Starr RH Jr. Early intervention and recovery among children with failure to thrive: follow-up at age 8. Pediatrics 2007;120:59-69. 